\section{Corneal perspectives}

\section{By Benjamin Boettner, Associate Editor}

Two independent findings could increase the availability of tissue for corneal transplants. A University of California, San Diego-led team has created a new source of limbal stem cells-which are needed for clear vision-by expressing PAX6 in skin epithelial cells, while a Harvard Medical School-led group showed that the ATP-binding cassette transporter ABCB5 is a marker for selection of limbal stem cells with enhanced regenerative potential. ${ }^{1,2}$

For the UCSD team, the therapeutic potential of the method might rest on whether it can produce a sufficiently homogeneous population of limbal stem cells for clinical use and find a clinically viable way to increase PAX6 (paired box 6) expression. The Harvard team has licensed an ABCB5 (ATP-binding cassette sub-family B (MDR/TAP) member 5)specific antibody to two stem cell companies-Rheacell GmbH \& Co. KG and Ticeba GmbH-that will develop it for clinical use. The terms of the deals were not disclosed.

Clear vision depends on a transparent layer of corneal epithelial cells that derive from limbal stem cells. Deficiency in those cells is a leading cause of blindness, and in most cases the only treatment is to replace them by transplantation. ${ }^{3,4}$
However, to be successful, the transplant requires sufficient numbers of limbal stem cells capable of sparking regeneration. Often these can be obtained from healthy cells in the contralateral, unaffected eye, which provides an autologous supply. But that option does not exist when both eyes are affected.

Under healthy conditions, the corneal surface continuously repopulates from limbal stem cells produced in the limbus, a niche-forming, narrow band of tissue surrounding the cornea. The cells migrate toward the cornea's center and differentiate into transparent, functional corneal epithelial cells. Corneas unable to supply sufficient limbal stem cells have an opaque, skin epithelium-like morphology.

Although faulty limbal stem cell generation has been known for some time to be a cause of corneal disease, how these cells differentiate and what factors control the process have not been clear.

\section{Skin in the game}

Kang Zhang and colleagues at UCSD partnered with researchers at Sun Yat-sen University, Sichuan University and Guangzhou KangRui Biological Pharmaceutical Technology Co. Ltd. to pinpoint the molecular drivers of limbal stem cell development with the long-term goal of creating a usable supply of cells for transplantation.

Zhang is a professor of ophthalmology at the Shiley Eye Center at the University of California, San Diego.

As a first step, the researchers cultured donor human limbal stem cells obtained postmortem and found the right conditions to produce an expanded, homogeneous population of cells. By modifying the culture conditions, they induced the cells to differentiate into corneal epithelial cells.

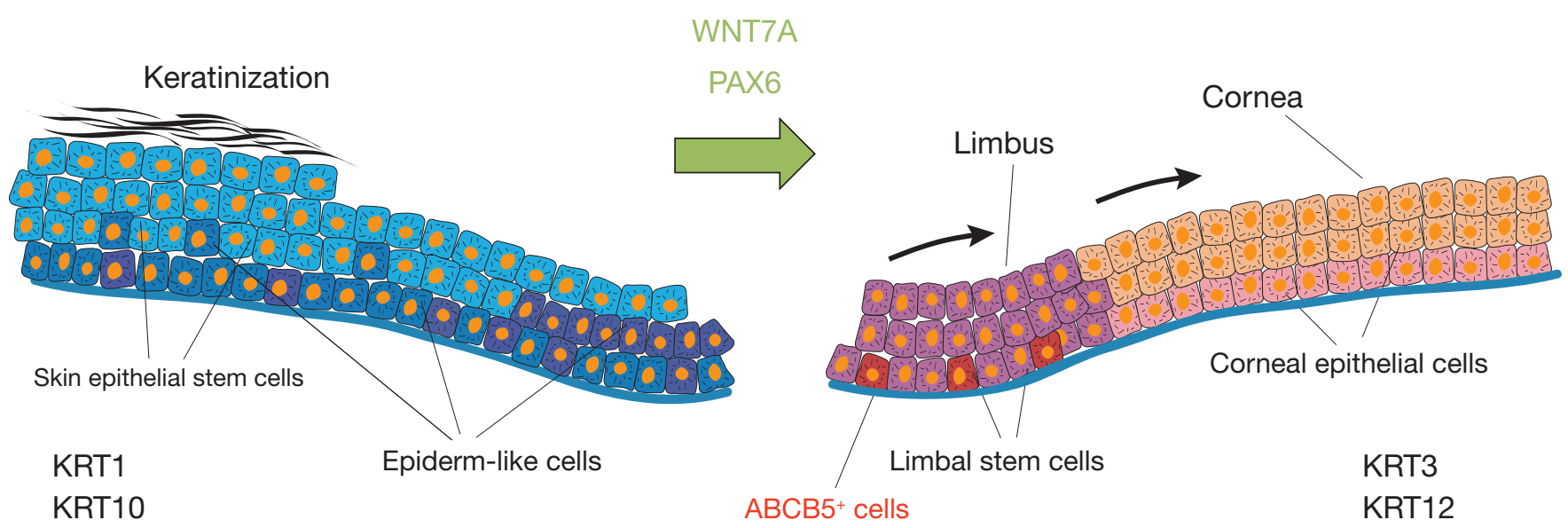

Figure 1. PAX6 drives differentiation of skin epithelial stem cells to limbal stem cells. Two new approaches that promote regenerative therapies for corneal transplants have been reported in Nature., In one study, researchers noted that wingless-type MMTV integration site family member 7a (WNT7A) activates paired box 6 (PAX6) to drive the conversion of skin epithelial stem cells to limbal stem cells that produce a healthy, clear cornea. ${ }^{1}$ Skin epithelial cells engineered to express $P A X 6$ established a healthy cornea after transplantation into a rabbit model of corneal disease. In the second study, researchers identified ATP-binding cassette sub-family $B$ (MDR/TAP) member 5 $(A B C B 5)$ as a marker for selection of limbal stem cells with high regenerative potential. ${ }^{2}$ Loss of all three genes results in the transition of a transparent corneal epithelial morphology with keratin 3 (KRT3; CK3) and KRT12 expression to an opaque skin epithelial morphology with KRT1 and KRT10 expression. 
Next, the team compared the genomewide expression profiles of limbal stem cells, corneal stem cells and skin epithelial stem cells from donor skin biopsies. The researchers identified two proteinswingless-type MMTV integration site family member 7a (WNT7A) and PAX6-that were expressed at significantly higher levels in limbal and corneal stem cells than in skin epithelial stem cells.

In addition, the researchers showed that in the limbal stem cells, WNT7A acts upstream of PAX6 and stimulates its expression via frizzled homolog 5 (FZD5), a receptor for WNT proteins.

WNT7A is a secreted morphogen involved in developmental and pathogenic WNT signaling. PAX6 is a transcription factor that controls the fate and differentiation of various eye tissues.

RNAi-mediated knockdown of WNT7A or PAX6 induced human limbal stem cells to transition from a corneal to a skin epithelial morphology. The WNT7A and PAX6 knockdown cells also had lower expression of corneal keratin 3 (KRT3; CK3) and KRT12 and greater expression of skin epithelial KRT1 and KRT10 than wild-type limbal cells (see Figure 1, "PAX6 drives differentiation of skin epithelial stem cells to limbal stem cells").

Conversely, overexpression of PAX6 caused skin epithelial stem cells to revert to a limbal stem cell phenotype. The team concluded that skin epithelial cells can be reprogrammed to produce limbal stem cells by the addition of that single factor.

Finally, the team showed that rabbit skin epithelial cells engineered to express Pax6 could re-establish a healthy cornea after transplantation into a rabbit model of limbal stem cell deficiency.

Results were published in Nature.

Zhang told SciBX that his team is now working on a PAX6-specific gene therapy and is testing WNT7A agonists as an alternative for reprogramming human skin epithelial cells to limbal stem cells.

UCSD has filed a patent covering the findings; it is available for licensing.

\section{Conversion considerations}

Because skin epithelial stem cells are far more abundant than limbal stem cells and are accessible in patients with bilateral disease, the findings suggest that skin-derived cells could provide a readily available source for limbal stem cell grafts. But it is still too soon to know how homogeneous the cultures are and what tools could reliably convert the approach to a viable system for clinical use.

Ruslan Semechkin, CSO of International Stem Cell Corp., told SciBX, "The study provides the first direct evidence demonstrating a functional connection between PAX6 and WNT7A and suggests that defects in the WNT7A-PAX6 axis are likely to be responsible for metaplastic conversion of corneal cells to skin epidermal-like cells in corneal diseases in humans. It points to a new strategy for treating corneal-surface diseases."

Michele De Luca agreed but said, "For a viable gene therapy approach with PAX6, it will have to be shown that skin epithelial stem cells can be reprogrammed in a safe way-for instance, under a minimal endogenous PAX6 promoter-and that indeed a bona fide limb stem cell population is stably generated."

According to Sophie Deng, previous studies in mice showed that skin epithelial cells can be converted to limbal stem cells when treated with corneal supernatants. "However, this is the first demonstration that human skin epithelial cells maintain the same plasticity," she said.

In addition, she said, because PAX6 regulates differentiation in several eye tissues, the team will need to assess the heterogeneity of the limbal stem cells produced by this system, in particular if the researchers plan to pursue gene therapy.

However, she added, "Skin epithelial stem cells will likely be a better source for transplantable limbal stem cells than induced pluripotent stem cells as they require less manipulation. Patients with bilateral corneal diseases could greatly benefit from an alternative autologous cell source."

De Luca is a professor of biochemistry and director of the Stefano Ferrari Center for Regenerative Medicine at the University of Modena and Reggio Emilia. Deng is an associate professor of ophthalmology and director of the Corneal Cell Biology Laboratory at the Jules Stein Eye Institute at the University of California, Los Angeles.

Although PAX6 is more likely to be used in creating a regenerative therapy, WNT7A could be developed directly as a therapeutic.

However, Semechkin said that option might not be straightforward. "WNT proteins are highly hydrophobic, and so far all efforts to generate fully active WNT variants have failed," he said. But he added that WNT7A analogs have shown proof-of-concept in animal models of muscular dystrophy and thus could be explored for corneal disorders.

Deng suggested that small molecule screens could be performed to identify compounds that facilitate or enable conversion of skin epithelial stem cells to limbal stem cells.

But according to De Luca, PAX6 would be a better target to focus on because WNT7A might have to be continuously supplied in patients with chronic corneal disease to prevent limbal stem cells from reverting to skin epithelial stem cells.

Fate Therapeutics Inc. has the biologic FT301, a WNT7A analog, in preclinical testing for muscular dystrophy.

\section{Mark the limbal stem cells}

In a second study, a team from Harvard Medical School and Brigham and Women's Hospital wanted to find a marker to help select limbal stem cells with high regenerative potential for use in transplants.

Because stem cells in other tissues are marked by $A B C B 5$ expression and the protein has been associated with stem cell functions, the team thought the protein could also identify limbal stem cells and might play a vital role in corneal homeostasis. 


\section{ANALYSIS}

\section{TARGETS \& MECHANISMS}

ABCB5 is a member of the ATP-binding cassette superfamily of transporters that shuttles small molecules and drugs across the plasma membrane.

Using histological studies in mice, the team found that $A b c b 5$ is specifically expressed in the limbus and limbal stem cells and that

"Given the observations by [the UCSD group] and the finding that $A B C B 5$ helps amplify a PAX6+ limbal stem cell population, we would also like to test whether ABCB5 selection could enhance the conversion of skin epithelial stem cells to limbal stem cells."

- Markus Frank, Harvard Medical School

compared with no alteration.

To determine if Abcb5 could be used to select a subpopulation of limbal stem cells with high regenerative potential, the researchers developed a mAb against Abcb5 and used it to segregate $\mathrm{Abcb} 5^{+}$from Abcb5 limbal stem cells from mice. Segregated or unsegregated cells were then introduced into a mouse model of limbal stem cell deficiency.

$\mathrm{Abcb}^{+}$grafts yielded clear corneas within 5 weeks that had normal histology and $47 \%$ higher $K r t 12$ expression than corneas treated with unsegregated grafts. By contrast, Abcb5 $5^{-}$grafts yielded opaque corneas with no detectable $\mathrm{Krt12}$-expressing cells.

The team was led by Natasha Frank and Markus Frank, both assistant professors at Harvard Medical School. Natasha Frank is also an associate physician at Brigham and Women's Hospital; Markus Frank is an associate physician at Boston Children's Hospital.

\section{Learning the $\mathrm{ABC}$ of $\mathrm{ABCB} 5$}

The identification of ABCB5 as a marker for limbal stem cells with high regenerative potential could provide another tool for improving the success of corneal transplants. The proportion of functional limbal stem cells correlates closely with the clinical success of corneal transplants.

Natasha Frank told SciBX, “To date, no molecular marker suitable for prospective limbal stem cell isolation has been available. Thus, our findings represent a critical first step toward isolating a molecularly defined and pure adult stem cell population. Our lab is now further dissecting the biology of human $\mathrm{ABCB} 5^{+}$limbal stem cells."

Natasha and Markus Frank pointed out that the predominant use of ABCB5-selected cells would be in patients with unilateral limbal stem cell deficiency who can have cells harvested from the contralateral, healthy side. But Markus Frank added that they are exploring whether the marker might also help in allogeneic transplants for patients with bilateral disease by enabling selection of cells with high regenerative potential from donors or limbal stem cell cultures.
Deng said that because the ABCB5-specific antibody labels some other cells in the mouse limbus, additional markers could help produce an even more homogeneous population. She said that her lab has shown FZD7 maintains undifferentiated human limbal stem cells and thus could be combined with ABCB5 selection as an additional marker for limbal stem cells. ${ }^{5}$

OncoMed Pharmaceuticals Inc. has an anti-FZD7 antibody in Phase I trials for various cancers.

Markus Frank said, "Given the observations by [the UCSD group] and the finding that ABCB5 helps amplify a PAX6 ${ }^{+}$limbal stem cell population, we would also like to test whether ABCB5 selection could enhance the conversion of skin epithelial stem cells to limbal stem cells."

Brigham and Women's Hospital has filed a patent covering the findings and the anti-ABCB5 $\mathrm{mAb}$. The IP has been licensed to the German stem cell companies Rheacell and Ticeba, which will develop a GMP-grade antibody for use in initial clinical trials. Markus Frank is a scientific advisor at both companies.

Boettner, B. SciBX 7(32); doi:10.1038/scibx.2014.943

Published online Aug. 21, 2014

\section{REFERENCES}

1. Ouyang, H. et al. Nature; published online July 2, 2014; doi:10.1038/nature13465

Contact: Kang Zhang, University of California, San Diego, La Jolla, Calif. e-mail: kangzhang@ucsd.edu

2. Ksander, B.R. et al. Nature; published online July 2, 2014; doi:10.1038/nature13426

Contact: Natasha Y. Frank, Brigham and Women's Hospital and VA Boston Healthcare System, Boston, Mass.

e-mail: nfrank@partners.org

Contact: Markus H. Frank, Boston Children's Hospital, Boston, Mass.

e-mail: markus.frank@childrens.harvard.edu

3. Dua, H.S. et al. Eye (Lond.) 17, 877-885 (2003)

4. Rama, P. et al. N. Engl. J. Med. 363, 147-155 (2010)

5. Mei, H. et al. Stem Cells 32, 938-945 (2014)

COMPANIES AND INSTITUTIONS MENTIONED

Boston Children's Hospital, Boston, Mass. Brigham and Women's Hospital, Boston, Mass.

Fate Therapeutics Inc. (NASDAQ:FATE), San Diego, Calif. Guangzhou KangRui Biological Pharmaceutical Technology Co. Ltd., Guangzhou, China

Harvard Medical School, Boston, Mass.

International Stem Cell Corp. (OTCBB:ISCO), Carlsbad, Calif. Jules Stein Eye Institute at the University of California, Los Angeles, Calif.

OncoMed Pharmaceuticals Inc. (NASDAQ:OMED), Redwood City, Calif.

Rheacell GmbH \& Co. KG, Heidelberg, Germany Shiley Eye Center at the University of California, San Diego, La Jolla, Calif.

Sichuan University, Sichuan, China

Stefano Ferrari Center for Regenerative Medicine at the

University of Modena and Reggio Emilia, Modena, Italy

Sun Yat-sen University, Guangzhou, China

Ticeba GmbH, Heidelberg, Germany

University of California, San Diego, La Jolla, Calif. 